\title{
¿Cuál es la mejor opción de tratamiento quirúrgico en varones con síntomas de la vía urinaria inferior?
}

\section{What is the best surgical treatment option for male patients with lower urinary tract symptoms?}

\author{
Antonio Alcántara Montero, ${ }^{1}$ Carlos Müller Arteaga²
}

\section{Sr. Editor:}

Los síntomas de la vía urinaria inferior, provocados por hiperplasia benigna de próstata, son altamente prevalentes en varones de edad avanzada. El tratamiento quirúrgico se recomienda en pacientes que no están dispuestos a comenzar o continuar una terapia médica, varones que experimentan progresión de los síntomas de la vía urinaria inferior con tratamiento farmacológico y con síntomas de la vía urinaria inferior complicados, incluidos: retención urinaria recurrente, incontinencia por rebosamiento, infecciones recurrentes de la vía urinaria, cálculos en la vejiga, hematuria macroscópica recurrente por hiperplasia benigna de próstata y dilatación de la vía urinaria superior. Si bien las indicaciones están bien establecidas, en las últimas dos décadas se han visto incrementadas las técnicas quirúrgicas para el tratamiento de los síntomas ocasionados por el agrandamiento benigno de la próstata. Además de la resección transuretral de próstata, la enucleación endoscópica de la próstata y la vaporización fotoselectiva de la próstata son técnicas para tratar los síntomas debidos al agrandamiento benigno de la próstata. ${ }^{1}$ La cirugía temprana es benéfica para evitar las complicaciones. ${ }^{2}$ Llegados a este punto de partida surge una pregunta: ¿cómo elegir la mejor opción de tratamiento quirúrgico para un paciente con síntomas de la vía urinaria inferior?

\footnotetext{
${ }^{1}$ Especialista en Medicina Familiar y Comunitaria, Centro de Salud José María Álvarez, Don Benito, Badajoz, España. Miembro del Grupo de Trabajo de Urología (Nefrología y Vías Urinarias) de la Sociedad Española de Médicos de Atención Primaria (SEMERGEN).

${ }^{2}$ Especialista en Urología, Departamento de Urología, Complexo Hospitalario Universitario de Ourense, España.
}

Recibido: julio 2018

Aceptado: julio 2018

Correspondencia

Antonio Alcántara Montero a.alcantara.montero@hotmail.com

Este artículo debe citarse como Alcántara-Montero A, Müller Arteaga C. ¿Cuál es la mejor opción de tratamiento quirúrgico en varones con síntomas de la vía urinaria inferior? Rev Mex Urol. 2018 julio-agosto;78(4):334-346 DOI: https://doi.org/10.24245/revmexurol.v78i4.2367 
La seguridad y eficacia de estas técnicas han sido demostradas. La elección del tratamiento debe hacerse de manera individualizada y está determinada por los siguientes factores (Figura 1) $)^{3}$ :

1. Tamaño de la próstata. Para pacientes con volumen prostático $<100 \mathrm{~mL}$ la resección transuretral de próstata todavía se considera la técnica de referencia. Basado en la morbilidad perioperatoria y posoperatoria, la resección transuretral de próstata bipolar parece preferible a la resección transuretral de próstata monopolar. Sin embargo, la enucleación endoscópica de la próstata y vaporización fotoselectiva de la próstata también han arrojado resultados comparables con la resección transuretral de próstata en esta población. Para pacientes con volumen prostático $>80 \mathrm{~mL}$ existe una alta evidencia disponible para enucleación endoscópica de la próstata, tanto con láser de holmium (Ho-
LEP), como para la enucleación bipolar de la próstata. Aunque también se ha informado que la vaporización fotoselectiva de la próstata es efectiva en estos pacientes, el nivel de evidencia alto es limitado.

2. Comorbilidades del paciente. En pacientes que no pueden suspender el tratamiento con anticoagulantes, antiagregantes plaquetarios, o ambos, la vaporización fotoselectiva de la próstata ha sido reportada como un tratamiento seguro y efectivo.

3. Preferencias del paciente y voluntad de aceptar los efectos secundarios relacionados con el tratamiento. En este contexto, los efectos secundarios sexuales, como la eyaculación retrógrada y el riesgo potencial de disfunción eréctil, deben tenerse en cuenta. En varones que no están dispuestos a aceptar el riesgo de dichos efectos secundarios sexuales pueden considerarse opciones alternativas de tratamiento mí-

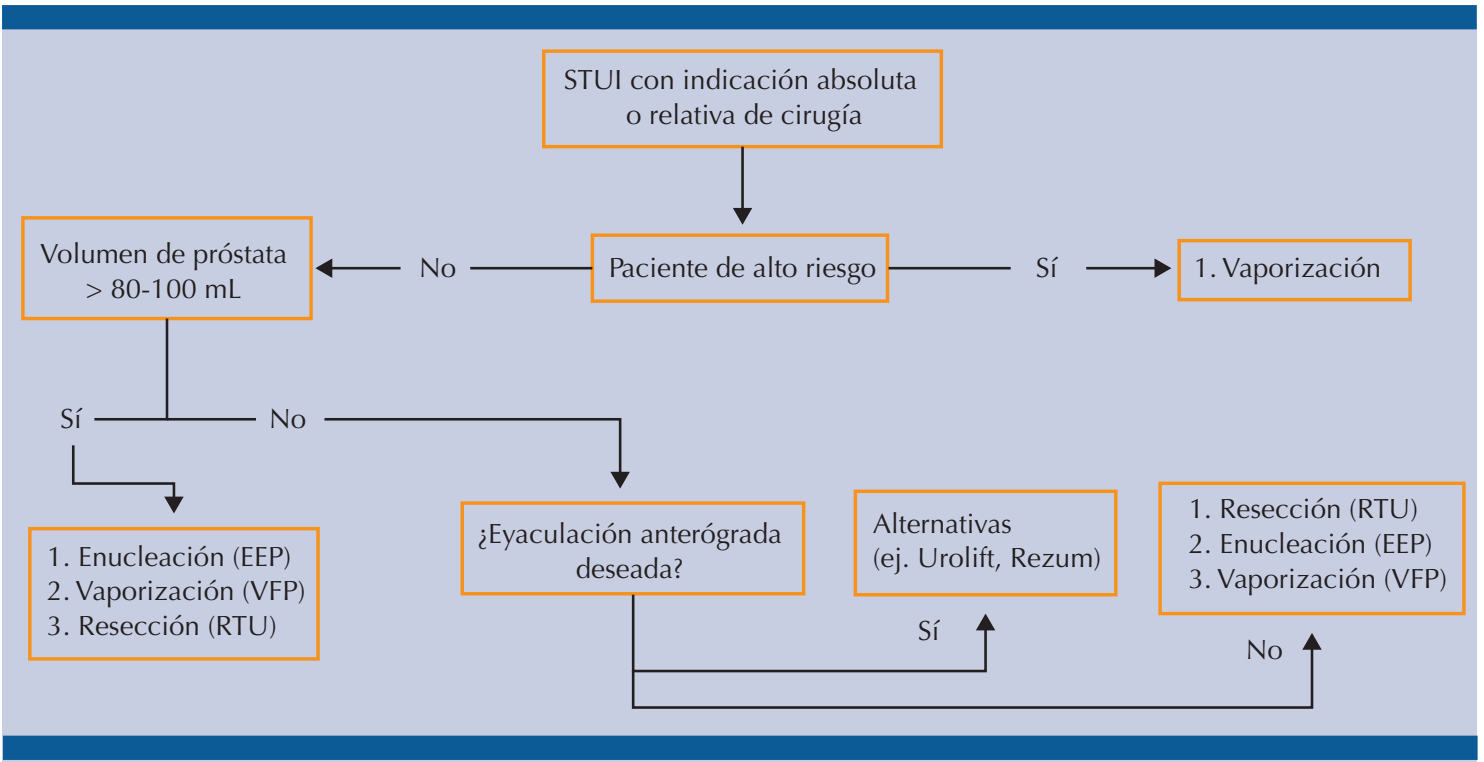

Figura 1: Opciones de tratamiento quirúrgico en pacientes con síntomas de la vía urinaria inferior.

EEP: enucleación endoscópica de la próstata; RTU: resección transuretral de próstata; STUI: síntomas de la vía urinaria inferior; VFP: vaporización fotoselectiva de la próstata.

Adaptado de Rieken $\mathrm{M}$ et al. 
nimamente invasivas como: elevación de la uretra prostática (Urolift) y ablación de la próstata mediante la energía natural almacenada en el vapor de agua (Rezum).

4. Experiencia del cirujano y disponibilidad de cada técnica en los centros.

En resumen, para los pacientes con síntomas de la vía urinaria inferior debidos al agrandamiento benigno de la próstata existe una gran variedad de opciones de tratamiento efectivas y mínimamente invasivas. La elección de dicho tratamiento debe estar adaptada a las características del paciente, comorbilidades y expectativas. Por todo ello, los pacientes necesitan estar informados de todas las alternativas terapéuticas. Al ofrecer un tratamiento individualizado pueden lograrse resultados quirúrgicos óptimos y calidad de vida posoperatoria.

\section{REFERENCIAS}

1. Gravas S, Cornu JN, Drake MJ, Gacci M, et al. EAU Guidelines on Management of Non-Neurogenic Male Lower Urinary Tract Symptoms (LUTS), incl. Benign Prostatic Obstruction (BPO). European Association of Urology (EAU). 2018. [en línea]. Direción URL: <http://uroweb.org/guideline/ treatment-of-non-neurogenic-male-luts/>. [Consulta: 2 de julio 2018].

2. Bachmann A, et al. Early surgical intervention is beneficial in avoiding complications in patients with male lower urinary tract symptoms. DOI: https://doi.org/10.1016/j. euf.2018.03.006

3. Rieken M, Kaplan SA. Enucleation, vaporization, and resection: How to choose the best surgical treatment option for a patient with male lower urinary tract symptoms. DOI: https://doi.org/10.1016/j.euf.2018.04.020

\section{AVISO IMPORTANTE}

La Revista Mexicana de Urología se convierte en una publicación solo digital, con todas las ventajas que los medios y dispositivos electrónicos ofrecen. Usted podrá revisar la información mediante el sitio web (www.revistamexicanadeurologia.org.mx) o descargando la app para Android o iPhone.

Para consultar el texto completo de los artículos deberá registrarse por una sola vez con su correo electrónico, crear una contraseña, indicar su nombre, apellidos y especialidad.

Esta información es indispensable para saber qué consulta y cuáles son sus intereses, y poder en el futuro inmediato satisfacer sus necesidades de información. 